Journal of Computer Sciences 1 (4 ): 477-481, 2005

ISSN 1549-3636

(C) Science Publications, 2005

\title{
The Use of Neural Network to Recognize the Parts of the Computer Motherboard
}

\author{
Abbas M. Ali, S.D.Gore and Musaab AL-Sarierah \\ Department of Information Technology, University of Al-Hussein Bin Talal, Maan, Jordan
}

\begin{abstract}
This study suggests a new approach of learning which utilizes the techniques of computer vision to recognize the parts inside the motherboard. The main thrust is to identify different parts of the motherboard using a Hopfield Neural Network. The outcome of the net is compared with the objects stored in the database. The proposed scheme is implemented using bottom -up approach, where steps like edge detection, spatial filtering, image masking..etc are performed in sequence. the scheme is simulated in MATLAB environment where the motherboard is treated as an image.
\end{abstract}

Key words: Image, edge detection, neural network, hopfield, digital image

\section{INTRODUCTION}

Computer imagining is a fascinating and exciting area to be involved in today ${ }^{[6]}$, where different steps such as translating the visual information in the form of digital images and processing a digital information, translating into another form e.g. sound or action and communication.

In this work we have taken the image of the motherboard since it is the fixed component inside a computer and trying to isolate the parts of the motherboard. Finally the isolated component then compared inside the database which contains also parts with associated meaning in a text this text will be in turn converted to action or voice.

This study deals with the design of Artificial Neural Network (ANN) to retrieve as well as processing the image of the motherboard. Because the image recognition involves many steps that can not be separated for each other like morphology, edge detection, canny transform. So we need to separate the image into many objects and therefore the database organized according to this idea. The object will be introduced to the (ANN) and this will be recognized according to this procedure. It is so important that the parts inside the image taken according to the coordinate dimensions as shown in Fig. 1.

The following sections will be discussed how the parts are separated, there are different approaches to parts dropout, some approaches use separate boundaries and then clean all other boundaries, we have used in our work determining the dimensions because of the parts are fixed. in computer vision applications the processed(output) images are for use by a computer, whereas in image processing applications the output images are for human consumption ${ }^{[6]}$.
Computer vision and image processing: Computer vision is a computer imagining where the application does not involve a human being in the visual loop, in other word, the image are examined and acted upon by a computer

The determining the parts inside the image of the motherboard it needs image analysis. Image analysis involves the examination of the image data to facilitate solving a vision problem. The image analysis process involves two other topics: feature extraction and pattern classification, the former is the process of acquiring higher level image information, such as shape, or color information and the later is the using of the higher level information and identifying objects within the image.

There are many applications and works has done in this fields, such as manufacture quality control, different areas within the medical community like diagnose skin turmors automatically identification of finger prints to DNA analysis, in US space program and department..etc ${ }^{[4]}$

Artificial Neural Network is an information processing system that has certain performance characteristics in common with biological Neural Networks ${ }^{[1]}$

Neural Networks have became a popular technique for pattern recognition, face detection ${ }^{[1,3-5]}$. They contain layers of processing elements, to process the input data coming from the image.

Many techniques are used in processing the image pattern processing like modular architectures, committee ensemble classification, complex learning algorithms, associative and compression networks and networks evolved or pruned with genetic algorithms. We shall discuss a Madaline neural networks in the next section as a neural network may be use in the same purpose. 


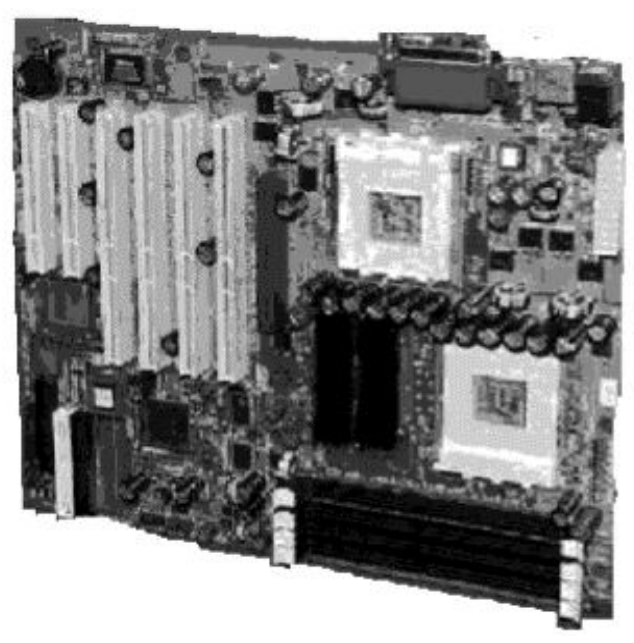

Fig. 1: The image of the motherboard

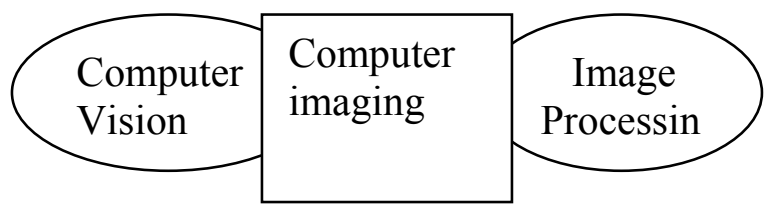

Fig. 2: The relation between computer vision and image processing

Layered neural networks: The Madalines of 1960s had adaptive first layers and fixed threshold functions for the second (output) layers ${ }^{[4,8,9]}$, the feed-forward neural nets of the 1980's have many layers, the back-propagation method for Rumelhart etal ${ }^{[4,10]}$ is perhaps one example of a multiplayer network. Fig. 3 below shows a three-layer feed forward adaption network to recognize the memory part and the CPU part

The back-propagation algorithm is one method for establishing desired responses for the internal layers or hidden layers, those layers which will acts of appearing the desired output.

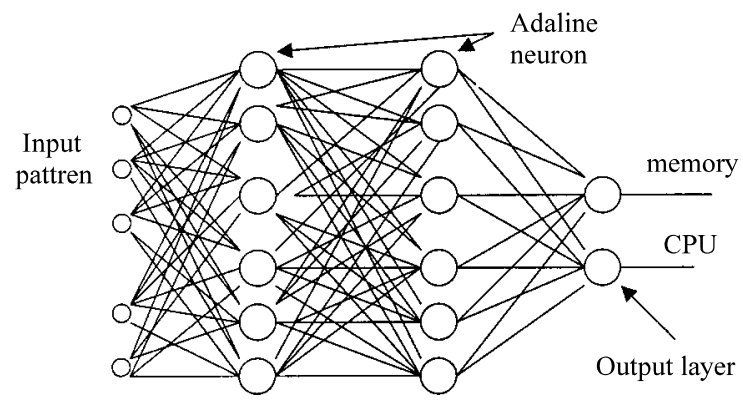

Fig. 3: Layered neural network for recognition of the parts

We denote for development of the algorithm for adapting layered networks of Adaline with hard limiting quantities. The algorithm is an extension of the original Madaline adaption rule ${ }^{[8,9]}$ is called Madaline rule II, MR II, the idea is to adapt the network to properly respond to the newest input pattern ${ }^{[4]}$.

When training the network to respond correctly to the various input patterns, the "golden rule" is give the responsibility to the neuron that can most easily assume it. Fig. 4. below shows the relation between the image picked by the camera and the Adalines

We have used hopfield neural network to recognize the object separated inside the image of the motherboard as in Fig. 5, the neural network is designed to look at windows of $150 \times 150$ pixels.

The neural network will compare the image with database stored image, these two sides of comparison images will be processed by canny transform algorithm to detect the edge inside the image and this will simplify the comparison more.

The architecture of motherboard parts detection: In this section we shall explain the architecture of this system where clarify the main steps used to reach the detection of the parts, first at all the data base contain already stored preprocessed image, with its name the

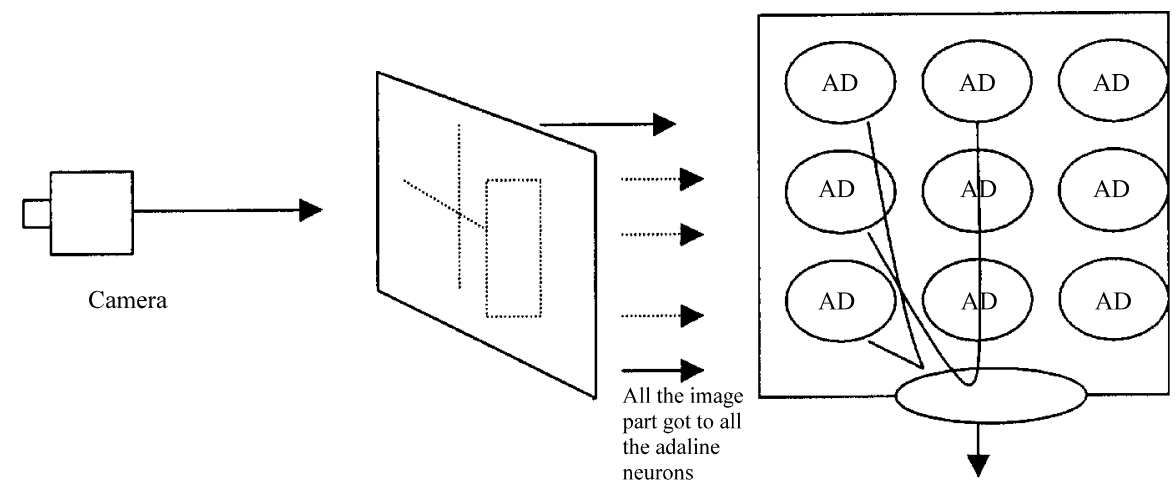

Fig. 4: The image entered to layered neural network 


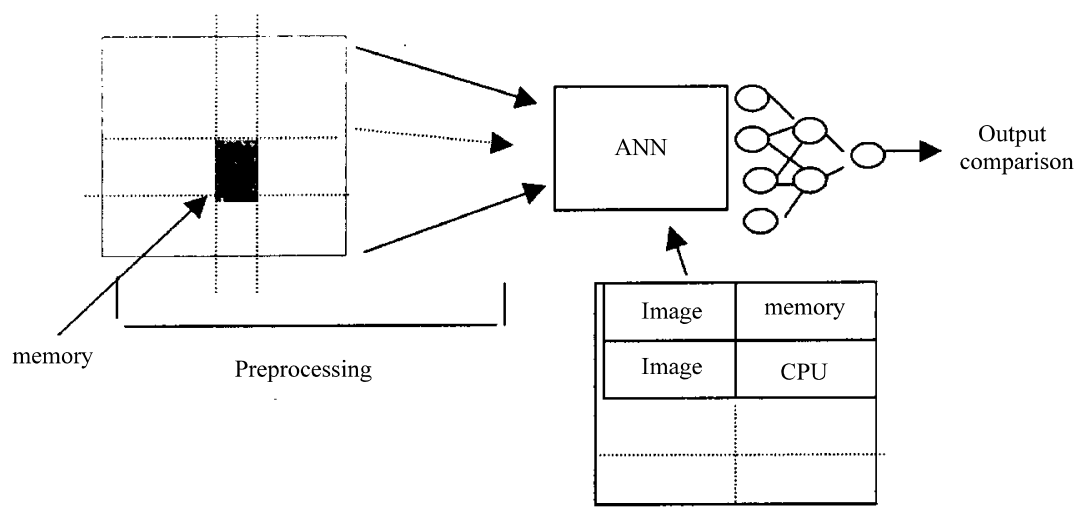

Fig. 5: Comparison of the image with the database image

Fig. 6 shows the main steps to process the image for the motherboard

The algorithm of the

START

. input images of the motherboard

. for each I inside the database

compare (I, X(0))

if yes then

disappear the associated explanation of the image

end for

if not found

there is some errors of recognition the image please try again to take another image end if

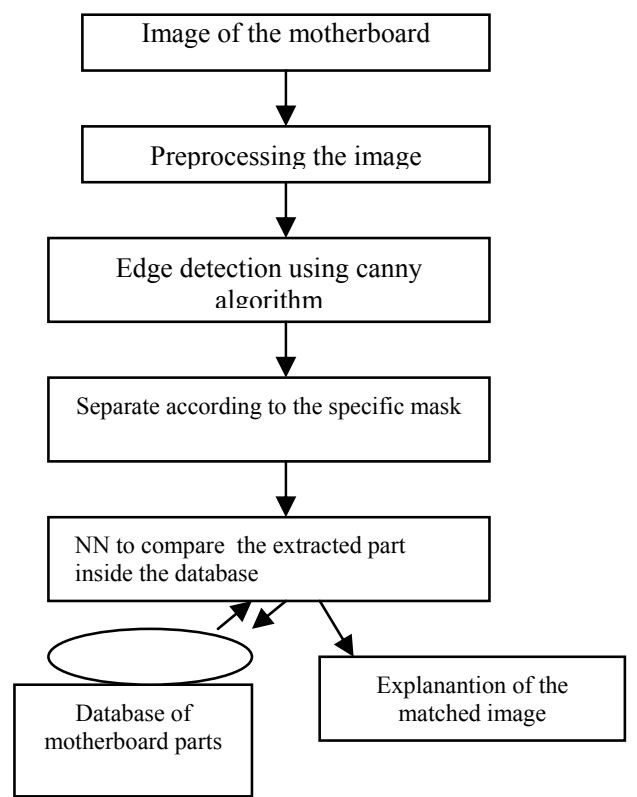

Fig. 6: The Algorithm and steps of image motherboard processing

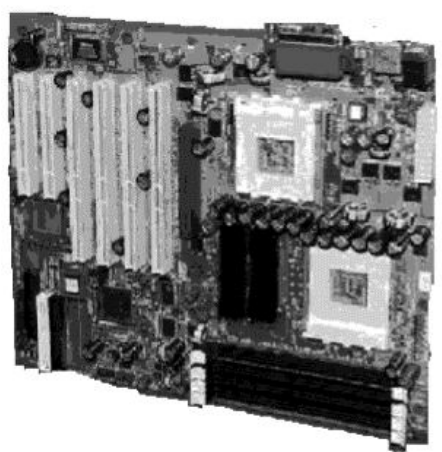

a) Original image

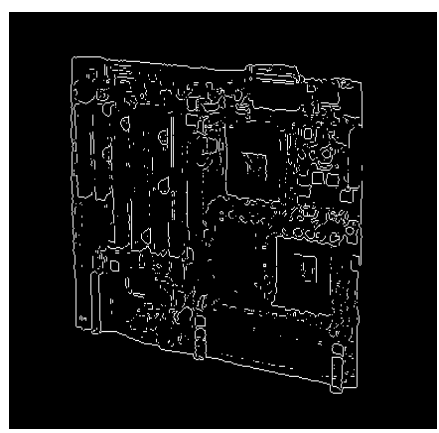

b) Sobel image

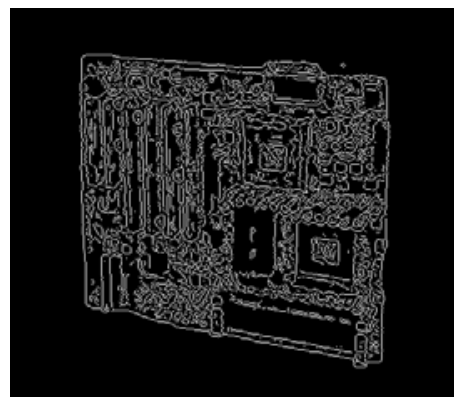

c) Canny Image 


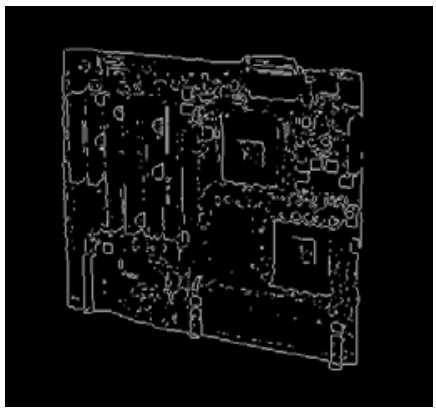

d) Prewitt image

Fig 7:

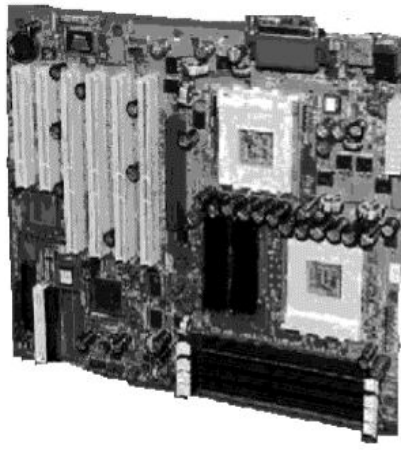

a) Original image

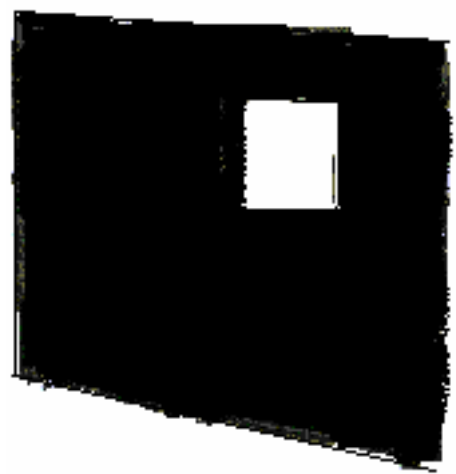

b) Square for And Mask

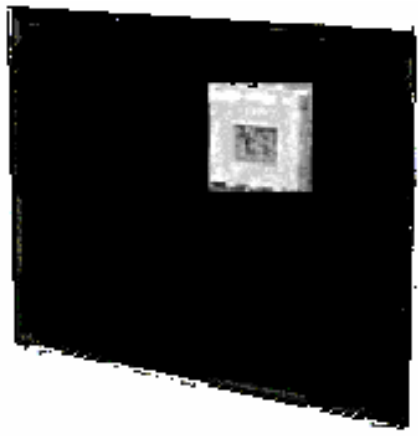

c) Resulting image (a) AND (b)

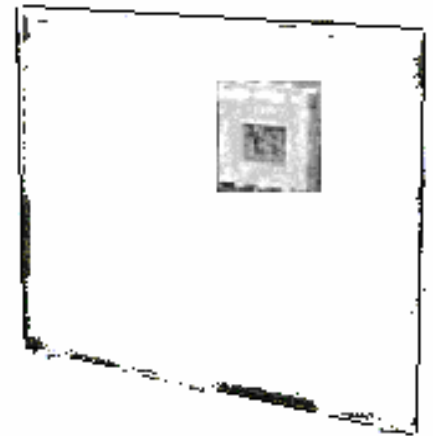

d) Square for OR mask

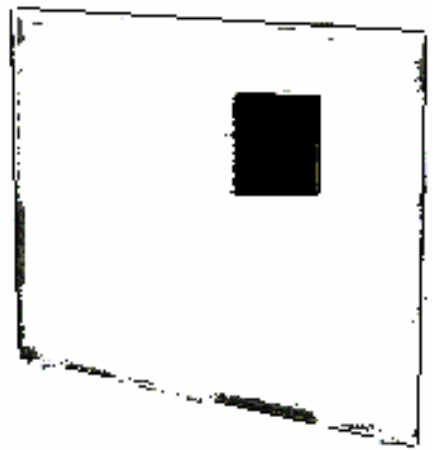

e) Resulting image (a) OR (d)

Fig. 7: The processing of the motherboard image to isolate the parts

\section{Processing the image}

Edge detection: this is the important thing to be done with the motherboard image, it is essentially locates major outlines in the image. It assigns each pixel on the line with a binary digit to set it out from the background many types of edge detection exist the Fig. 7 below shows some algorithm used for this purpose.

Here we have used a canny edge filtering, this is essential to detect the type of the motherboard used to explain for the user. since there are many types of motherboard, this is very useful when the image to compare with the inside stored image of the database.

Spatial filtering and image masking: Spatial filtering is typically done for noise removal or to perform some type of image enhancement ${ }^{[6]}$. The logical operation AND and OR are used to combine the information in two image such as for image extraction a region of interest from an image motherboard.

A white squared ANDed with an image will allow only the portion of the image coincident with the square to appear in the output image with the background turned a black and a black square OR ed with an image will allow only the part of the image corresponding to 
the black squared to appear in the output image but will turn the rest of the image white. the image masking is very essential to extract the parts in the motherboard image as in Fig. 7 below.

\section{CONCLUSIONS}

This can detect the parts of the motherboard from the image in a technique using some algorithms applied already but here will be in a useful way in learning field, explaining the images to the user using these methods, we think that it will be very useful to explain the parts inside the laboratory with out the administrator.

\section{REFERENCES}

1. Laurence Fauselt, 1994. Fundamentals of neural networks., prentice-Hall.

2. Howell, D., 2000. Getting to grips with graphics file format. Computer, Publishing issue.

3. Haykin, S., 1994. Neural networks; A comprehensive foundation, Prentice-Hall, New-Jersey, USA.
4. The computer society, 1988. Artificial neural systems. Computer Magazine, IEEE, 1988.

5. Kevin Curran, Xuelong and Neil Mc Caughley, 2005. The use of neural networks in real-time face detection. Science Publications, USA.

6. Scott, E. Umbaugh, 1998. Computer vision and image processing a practical approach using CVIP tools. MacGraw Hall.

7. Rafael C. Gonzalez and Richard E. Woods, 2002. Digital image processing. Prentice Hall.

8. Ridgway, W.C., 1962. An adaptive logic system with generalizing properties, Ph.D Thesis, Stanford Electronics Labs, Rep, 1556-1, Stanford University, Stanford, Calif.

9. Nilsson, N., 1965. Learning machines, McGraw-Hill, New York.

10. Rumelhart, D.E. and J.L. McClelland, 1986. Parallel Distrbuted Processing. MIT press, Cambridge, Mass, Vol: 1. 